\title{
The Use of Co-Design in Developing Physical Activity Interventions for Older Adults: A Scoping Review
}

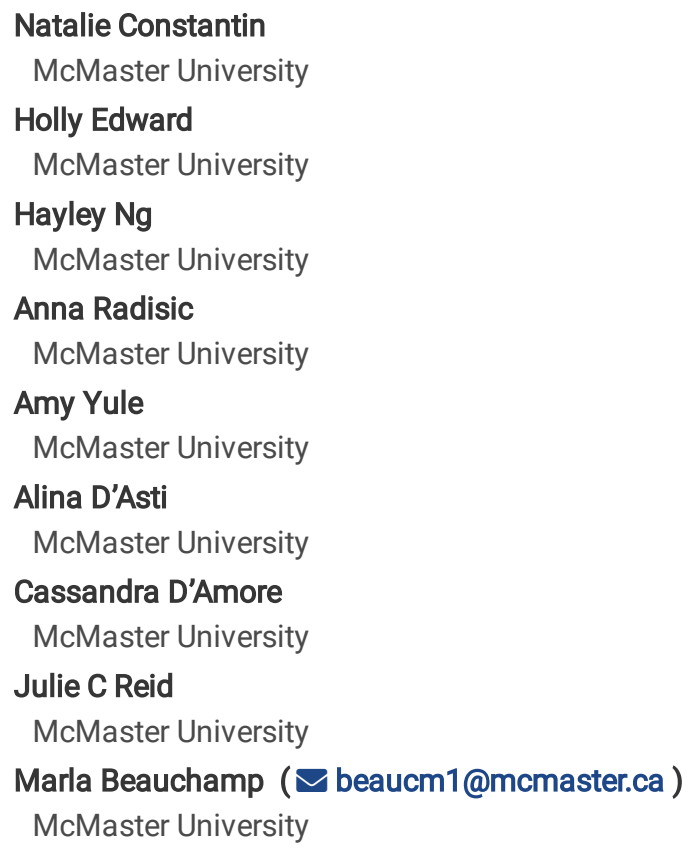

Keywords: aging, exercise, health promotion, participation, participatory research, seniors

Posted Date: February 3rd, 2022

DOI: https://doi.org/10.21203/rs.3.rs-1243879/v1

License: (c) (i) This work is licensed under a Creative Commons Attribution 4.0 International License. Read Full License 


\section{Abstract}

Background: Promoting physical activity (PA) participation in older adults is important for preserving quality of life and functional independence. Co-design has been shown to increase engagement of end-users in health-related policies and interventions. This scoping review aimed to examine how co-design has been used to develop PA interventions for older adults.

Methods: We searched MEDLINE, EMBASE, AMED, and CINAHL. Peer-reviewed primary research studies that met the following criteria were included: had at least one participant aged $\geq 60$ years involved in the co-design process and the intervention was delivered to individuals whose mean age was $\geq 60$, used co-design methodologies, and any form of PA. After duplicate removal, two or more independent reviewers completed title and abstract and full text screening. Data were extracted from the included studies according to study aims.

Results: Of the 29 included studies, 12 different terms were used to describe co-design with variable operational definitions that we consolidated into five proposed components. Fifteen studies engaged users in a consultative way, 13 studies using collaboration, and one study engaged end-users in consumer-control. No studies involved end-users in the dissemination phase. Further, no studies directly measured the effectiveness of the co-design process. Five categories of barriers and facilitators to co-design were identified including frameworks and methodologies, logistics, relationships, participation, and generalizability.

Conclusions: There is a large degree of variability in how co-design is used to develop PA interventions for older adults. Our findings can be used by researchers to improve rigor and standardization in this emerging field.

OSF registration: osf.io/vsw2m

\section{Background}

Co-design is an emerging methodology within healthcare research $(1,2)$. It aims to actively engage specific groups of individuals, such as endusers, to aid in the development of products or services through knowledge sharing (3). Co-designed initiatives promote patient-centered care by incorporating varying degrees of stakeholder input into the development or reform of health services while providing quality assurance (4). These initiatives are embodied in the mandates of organizations such as the Patient Centred Outcomes Research Institute (PCORI) in the United States, which advocates for clinician, patient, and end-user involvement throughout healthcare related research (5).

Physical activity (PA) participation across the lifespan is essential for maintaining functional independence and preventing chronic disease later in life (6-13). In this review, we define PA as any form of activity that results in the expenditure of energy (14). The World Health Organization (WHO) recommends that adults aged $\geq 65$ years complete 150-300 minutes of moderate-intensity aerobic activity or 75-150 minutes of vigorous-intensity aerobic activity per week in addition to two or more days of strength and balance training (15). Older adults who participate in regular PA are less likely to develop conditions such as cardiovascular disease, diabetes, and stroke (16). Furthermore, they are more likely to notice positive improvements in their mental health and quality of life (16). However, despite the known importance of PA, the WHO estimates that $1 / 4$ of adults globally do not meet the minimum recommended guidelines (17). Additionally, the WHO estimates approximately 3.2 million deaths per year are due to physical inactivity (17).

At the onset of the COVID-19 pandemic, leaders implemented stringent physical distancing measures to prevent the spread of the virus and protect vulnerable populations. While this has helped curb the spread of the virus in some communities, it has contributed to an increase in sedentary behaviour $(18,19)$. International research shows that the community lockdowns and physical distancing regulations due to the COVID-19 have drastically decreased physical activity levels in all age groups, including older adults (18-23).

Evaluations of the impact of patient and public involvement in research show that patients, communities, and researchers all benefit from codesign (24). A preliminary search focused on co-design methodologies and PA interventions in older adults identified one systematic review and one narrative literature review examining barriers and facilitators to end-user involvement $(25,26)$. Co-design is gaining popularity in health research and policy development, but a clear description of terminology, methodology, and evaluation tools is lacking (2). This scoping review maps the current state of the literature on the use of co-design for developing PA interventions for older adults and identifies gaps for future research. To our knowledge, this is the first review to summarize terminology and definitions that describe co-design, as well as map how and when end-users are involved throughout the process.

\section{Objectives}

The overall objective of this scoping review was to examine how co-design has been used to develop PA interventions for older adults. The specific aims of this review were to: 1) report the terminology and definitions that have been used to describe co-design in included studies, 2) 
describe what phases of the research process co-design has been used, 3) determine the levels of involvement of the end-users, 4) understand how the success and/or effectiveness of co-design has been measured, and 5) identify barriers and facilitators for the co-design process.

\section{Methods}

Full details of the study methodology are outlined in the study protocol (27). We followed standardized methodology when conducting this scoping review (28-30), and reported our work according to the Preferred Reporting Items for Systematic Reviews (PRISMA-ScR) Extension for scoping reviews (Additional File 1) (31). Our search strategy was developed in consultation with two health science research librarians (32). We searched 4 electronic databases from inception through Feb. 18, 2021, including OVID MEDLINE, EMBASE, AMED, and CINAHL. The full search strategy can be found in Additional File 2. Relevant reviews were retained for hand searching of reference lists. Two independent reviewers conducted all stages of screening (i.e., titles, abstracts, and full texts) and data extraction. Disagreements at any stage were resolved by consensus, or by a third reviewer where necessary. Agreement between reviewers was calculated at the title and abstract stage (33).

\section{Inclusion and Exclusion}

Criteria were aligned with the participant, concept, and context framework. We included: Participant - at least one participant aged $\geq 60$ years involved in co-design of an intervention for a target population whose mean age was $\geq 60$ years; Concept - co-design of a PA related intervention as defined in our introduction; Context - any clinical population or setting. We excluded studies that did not use co-design methodologies or involve PA. We also excluded grey literature, literature reviews, and non-English studies. We imported all citations into Covidence v.2576 c3a8578b (Veritas Health Innovation, Melbourne, Australia). The results of the search and the study inclusion process were reported in full in a PRISMA-ScR flow diagram in Additional File 3 (31).

\section{Data Extraction and Synthesis}

Co-design terminology and operational definitions were extracted verbatim from text. Operational definitions were analyzed, and repetitive concepts identified. Similar concepts were highlighted with the same colour. For example, concepts such "partnership", "collaboration", "working with" and "shared leadership" were grouped together under the "collaboration between researchers, older adults, and other relevant stakeholders" component. Studies were categorized according to the planning, conducting, and dissemination phases of involvement described by the Patient-Centered Outcomes Research Institute (PCORI) (34) and similarly by the consultation, collaboration, and consumer control levels of involvement outlined by Boote, Telford, and Cooper (35). Outcome measures used to examine the success (the accomplishment of research aims) and/or effectiveness (the extent of producing the desired result) of the co-design process were recorded. Authors were contacted by email when missing or additional information was required.

\section{Results}

Study inclusion

From four electronic databases, we identified 10,956 citations. An additional 8 citations were retrieved from manual searching of reference lists. We identified 29 papers that met our inclusion criteria. Reasons for exclusion are reported in our PRISMA flow chart (Additional File 3 ). A detailed list of all citations excluded on full-text examination and reasons for exclusion can be found in Additional File 4. Agreement between reviewers for title and abstract screening was between 0.84 and 1 .

Characteristics of included studies

Included studies were published between 2000 and 2021, with $82 \%$ of the studies published since 2016. Multiple countries of origin were represented across included studies such as the United Kingdom (17\%), United States (14\%), and the Netherlands (10\%). Most studies (90\%) were set in the community, followed by long-term care or retirement homes (7\%), and then hospital (3\%). In terms of study design, $76 \%$ were mixed methods, $10 \%$ randomized controlled trials, $7 \%$ protocols, $3 \%$ case studies, and $3 \%$ cross-sectional design. Additional File 5 summarizes the characteristics of our included studies.

Terminology and Operational Definitions of Co-Design

We identified 12 different terms to describe the process of co-design. Figure 1 shows the frequency of each term. Twenty-six (90\%) studies provided operational definitions (Additional File 6), and 3 studies did not. Thematic analysis of the 26 operational definitions revealed repetitive concepts that were categorized into 10 themes that were further grouped into 5 proposed components of co-design as shown in Table 1. From this analysis, our proposed standardized definition for co-design is a user-centered approach involving collaboration between researchers, end-users, and other relevant stakeholders who are actively engaged throughout a process of iteration and continuous reflection to create an intervention tailored to the specific needs of the target population. 
We systematically identified whether the proposed components of our co-design operational definition were carried out in each study's methodology. Twelve studies included a user-centered approach, 17 demonstrated collaboration between researchers, older adults, and other relevant stakeholders, 11 tailored their interventions to the specific needs of the target population, 10 actively involved end-users throughout the study, and 5 demonstrated iteration or continuous reflection.

Figure 1. Use of Co-Design Terminology in Included Studies

Table 1

Thematic Analysis of Co-Design Operational Definitions

\begin{tabular}{|c|c|c|c|c|c|}
\hline \multirow[t]{3}{*}{ Terminology } & \multicolumn{5}{|c|}{ Proposed Components of Co-Design } \\
\hline & \multirow{2}{*}{$\begin{array}{l}\text { User- } \\
\text { centered } \\
\text { approach } \\
(38-51)\end{array}$} & \multirow{2}{*}{$\begin{array}{l}\text { Collaboration between } \\
\text { researchers, older adults, } \\
\text { and other relevant } \\
\text { stakeholders } \\
(39,41,42,44-46,48,50- \\
61)\end{array}$} & \multirow[t]{2}{*}{$\begin{array}{l}\text { Tailored to specific needs } \\
(38,39,46,47,50,53,55,57,58,62,63)\end{array}$} & \multirow{2}{*}{$\begin{array}{l}\text { Active } \\
\text { involvement } \\
\text { throughout } \\
(39,43,45,48- \\
50,58-61,64)\end{array}$} & \multirow[t]{2}{*}{$\begin{array}{l}\text { Iteration and } \\
\text { continuous } \\
\text { reflection } \\
(38,49,50,56,58)\end{array}$} \\
\hline & & & & & \\
\hline $\begin{array}{l}\text { Co-Design } \\
(39,40,50,65)\end{array}$ & $(39,40,50)$ & $(39,50)$ & $(39,50)$ & $(39,50)$ & (50) \\
\hline $\begin{array}{l}\text { Co-Creation }(13,28,30- \\
33,37,39,42,43,54)\end{array}$ & $\begin{array}{l}(39,41- \\
45,49,51)\end{array}$ & $(39,41,42,44,45,51,54,55)$ & $(39,55)$ & $(39,43,45,49)$ & $(49)$ \\
\hline $\begin{array}{l}\text { Action Research } \\
(41,42,51,58,64,67,68)\end{array}$ & & $(41,42,51,58)$ & $(58)$ & $(58,64)$ & $(58)$ \\
\hline $\begin{array}{l}\text { Participatory Design } \\
(38,45,48,57)\end{array}$ & $(38,45,48)$ & $(45,48,57)$ & $(38,57)$ & $(45,48)$ & \\
\hline $\begin{array}{l}\text { Community-Based } \\
\text { Participatory } \\
\text { Research/Participatory } \\
\text { Research }(46,59,60,63)\end{array}$ & $(46)$ & $(46)$ & $(46,59,60)$ & $(59,60)$ & \\
\hline $\begin{array}{l}\text { User-Centered Design } \\
(38,47,49)\end{array}$ & $(38,47,49)$ & & $(38,47)$ & & $(38,49)$ \\
\hline $\begin{array}{l}\text { Participatory Action } \\
\text { Research }(53,56)\end{array}$ & & $(53,56)$ & $(53)$ & & $(56)$ \\
\hline $\begin{array}{l}\text { Integrated Knowledge } \\
\text { Translation (52) }\end{array}$ & & $(52)$ & & & \\
\hline $\begin{array}{l}\text { Qualitative } \\
\text { Methodology (62) }\end{array}$ & & & $(62)$ & & \\
\hline User-involvement (43) & (43) & & & $(43)$ & \\
\hline Co-learning (64) & & & & $(64)$ & \\
\hline $\begin{array}{l}\text { Stakeholder } \\
\text { involvement }\end{array}$ & & & & (61) & \\
\hline (61) & & & & & \\
\hline Total & 12 & 17 & 11 & 10 & 5 \\
\hline
\end{tabular}

Use of Co-Design in the Phases of Research

Figure 2a shows the distribution of studies according to the phase of research in which end-users were involved. Nine studies utilized codesign in more than one phase, such as both planning and conducting.

Figure 2a. Distribution of studies according to phase of research in which end-users were involved.

Determine the levels of involvement of the end-users in the included studies

The levels of involvement of end-users progress from consultation to collaboration to consumer control the highest level involvement. Most end-users were involved at either the consultation (52\%) or collaboration (45\%) level in the included studies. Figure $2 \mathrm{~b}$ summarizes the different levels of involvement, the number of studies within each level, and the methods used within each level. 
Figure $2 \mathrm{~b}$. Levels of involvement of end-users in the co-design process.

Measuring Success and/or Effectiveness of Co-Design

None of the included studies described any type of process to evaluate the success and/or effectiveness of the co-design process itself. However, some studies evaluated the success and/or effectiveness of the co-design process through indirect methods, such as measuring participant satisfaction of the PA intervention itself $(n=4)$, intervention adherence measures $(n=6)$, and by assessing changes in PA performance and levels of PA $(n=5)$ (Table 2). Of the 15 studies that indirectly evaluated co-design, all reported positive trial outcomes that they attribute to the use of a co-design process.

Table 2

Outcome Measures used to Examine the Success and/or Effectiveness of Co-Design

\section{Indirect Methods}

\section{Participant Satisfaction}

-Gaming Experience Questionnaire (40)

-Fall Prevention Program Satisfaction Questionnaire (67)

-Program and Engagement Satisfaction Surveys $(54,61)$
Adherence

-Physical Activity Adherence Questionnaire (40)

-Attendance recorded $(54,58,61)$

-Recorded the number of days or time the product was used $(66,69)$

\section{PA Measures}

-Functional Capacity measured by The Senior Fitness Test (40)

-Fall Prevention Behaviors Questionnaire to assess five areas: 1) fall prevention practices, 2) regular vision assessment, 3) medication use, 4) exercise and 5) home environment (67)

-Physical Performance Test (PPT) to assess upper body muscle strength, lower body muscle strength, balance, and balance and gait (67)

-Recorded total steps/day (66)

-Amount of PA via accelerometer measurements $(59,60)$

-Measured physical fitness using handgrip strength, chair stand, 2-min step, back scratch, sit and reach, and flamingo balance test (59,60)

-Short Physical Performance Battery (SPPB) measures physical functioning using gait speed, standing balance, and lower leg strength (69)

Barriers and Facilitators Associated with Co-Design

We grouped author-reported barriers and facilitators into 5 categories, including framework and methodologies (any theoretical frameworks or principles used to formulate the co-design process and/or study methodology), logistics (details surrounding how the co-design process was organized and executed), relationships (dynamics among participants, and between participants and researchers), participation (participant engagement in the co-design process and the efforts made by researchers to increase engagement), and generalizability (aspects of the codesign process that help to make the intervention more applicable to the target population) (Table 3). 
Table 3

Barriers and Facilitators Associated with Co-Design

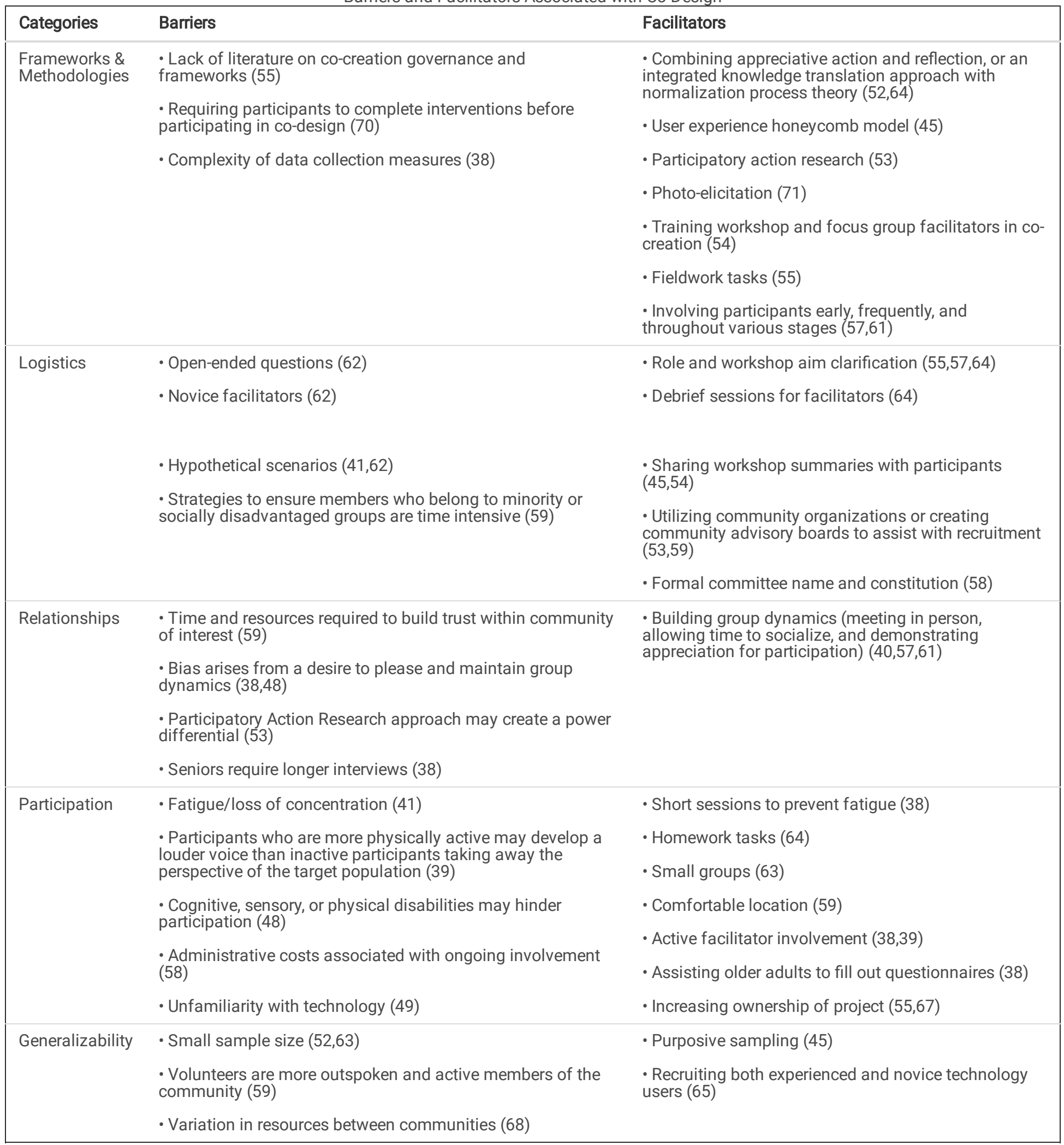

\section{Discussion}

This is the first scoping review to map the body of existing literature on co-design and PA in older adults, and to summarize how and when codesign is implemented. We identified 29 unique studies that reported on the use of co-design to create PA interventions for older adults. Three key findings emerged from the review process. First, there is substantial variability in the terminology and operational definitions used to describe co-design and we propose a standardized definition based on common elements in the literature. Next, most studies used co-design during the conducting phase of research, less in the planning phase, and none involved end-users during the dissemination phase. Lastly, no 
studies directly evaluated the success or effectiveness of their co-design approach. The findings of our review have practical implications for the design of PA interventions in older adults.

Our study identified substantial variability in terminology and operational definitions amongst studies. The lack of a single consistent conceptualization of co-design is not unique to research in geriatric health promotion. It has been recognized as a challenge in other health areas of co-design research in terms of developing a comprehensive search strategy and trying to synthesize the literature in order to advance this area of knowledge $(2,72,73)$. Although we could not recommend a single unifying term, we propose a unifying definition that incorporates common thematic elements from the literature. Based on our findings, we define co-design as a user-centered approach involving collaboration between researchers, end-users, and other relevant stakeholders who are actively engaged throughout a process of iteration and continuous reflection to create an intervention tailored to the specific needs of the target population. Interestingly, while only one study included all components of our proposed definition in their operational definition, 21 studies included at least four of the 5 key components in their methods.

With respect to phases of involvement, most studies used co-design in the conducting phase, but none engaged end-users in the dissemination process. This is a noteworthy finding, as experts recommend the use of targeted dissemination to ensure maximal uptake (24,25,35). Engaging end-users in dissemination may lead to more meaningful engagement of a wider range of people in the community, which could positively influence the uptake of evidence. Only one study involved end-users in consumer control. End-users should be involved throughout all phases of research to increase ownership of the findings among members of the public, who may then be more likely to share them within their social and community networks (35). Potential reasons for the lack of higher levels of involvement include lack of funding, time and resources, and lack of implementation from healthcare staff, as has been reported by Brett et al., Donetto et al., and Baldwin et al. (4,24,25).

Studies included in our review evaluated the co-design process indirectly by assessing outcomes downstream of the co-design process, such as the success of the PA intervention created during the co-design process, including PA, adherence, and participant satisfaction measures. These methods of evaluation are helpful to assess intervention adherence and to gauge participant satisfaction with the intervention or product, however, they do not allow evaluation of the co-design process itself. Despite all studies reporting positive outcomes of the PA intervention themselves, no study evaluated the success and/or effectiveness of the co-design process using direct methods, such as qualitatively assessing whether participants' views were accurately represented in the final intervention. A possible reason for this is the observable gap in the literature may be related to a lack of standardized ways to evaluate the co-design process $(2,36)$. Leask et al. recommends that evaluation be embedded throughout the phases of development to ensure that the intervention is representative of endusers' ideas and tailored to their specific needs and circumstance (36). Esmail and colleagues make similar recommendations and further suggest using external evaluators to minimize bias (74). Additional strategies could include member checking or respondent validation (75,76). Another approach is to conduct a process evaluation of the intervention through assessment of facilitators and barriers of implementation, fidelity, and reach (77).

Future research and implications

The field of co-design lacks a systematic framework to develop rigorous public health interventions and evaluate their efficacy and impact on a larger scale (36). Leask et al. aimed to outline recommendations and key elements for the application and evaluation of co-created public health interventions using existing frameworks and methodologies and suggest models for increasing the scale of interventions to a population level (36). We propose using common terminology and the operational definition proposed by our group to adopt a common language in this area of research. We recommend that future studies consider reporting their co-design interventions according to the guidelines by Leask et al. to improve the interpretation, replicability, and to guide the design of new studies (36).

Our review further highlights the barriers and facilitators associated with the co-design process and supports existing literature in this area (25). The barriers and facilitators summarized in this review can be used by researchers alongside recommendations by Leask et al. to design, implement, and evaluate co-designed interventions.

\section{Strengths and limitations}

Our study has some limitations. We were only able to include studies published in English for feasibility. We recognize that important studies may be published in other languages that could contribute further to this review and our understanding of the co-design literature, however given the breadth of included studies, we feel we were able to retrieve a representative sample of the literature. Lastly, our search strategy did not include grey literature, however, there are no central sources for grey literature leading to challenges in locating relevant citations and a high probability of selection bias.

Our study also has important strengths. We developed a comprehensive search strategy that was peer-reviewed by two health research librarians. We included a large number of studies from various countries, which contributes to our understanding of co-designed PA 
interventions on a global scale. Similarly, we included a range of study designs, which allowed us to provide a more comprehensive summary of the existing evidence base. We also used an established scoping review protocol and registered our study on Open Science Framework to limit publication bias.

\section{Conclusion}

Co-design is a growing and important area of research with substantial heterogeneity. This review mapped the co-design process for PA interventions in older adults, identifying gaps in when co-design is used and the level of involvement of end-users. Based on the included studies, we have suggested a standard definition of co-design for researchers to use moving forwards. Increasing the use of more standardized co-design methods presents an untapped potential for improving PA behaviour interventions. This review can help inform future co-designed interventions in their design and involvement of end-users to enhance the rigor and success of the process.

\section{Abbreviations}

\section{WHO}

World Health Organization

PA

physical activity

JBI

Joanna Briggs Institute

PRISMA

Preferred Reporting Items for Systematic Reviews

PCORI

Patient-Centered Outcomes Research Institute

AMED

Allied and Complementary Medicine database

CINAHL

Cumulative Index to Nursing and Allied Health Professionals

PRISMA-ScR

Preferred Reporting Items for Systematic Reviews and Meta-Analyses Extension for Scoping Review

\section{Declarations}

Ethics approval and consent to participate - Not applicable as this is a scoping review.

Consent for publication - Not applicable.

Availability of data and materials - Not applicable as this is a scoping review and therefore included no primary data collection.

Competing interests - The authors declare that they have no competing interests.

Funding - Marla Beauchamp holds a Tier 2 Canada Research Chair in Mobility, Aging, and Chronic Disease. Non-financial resources were shared to the authors by McMaster University. McMaster University provided access to databases and information specialists who provided insight on the topic and the research process.

Authors' contributions - NC, HE, and HN developed the search strategies for the electronic database. NC, HE, HN, MB, JCR, and CD developed the study methodology. NC, HE, HN, AD, AY, and AR all participated in title and abstract screening, full text screening, and data extraction. NC, $\mathrm{HE}$, and $\mathrm{HN}$ were major contributors in writing the manuscript. $\mathrm{MB}, \mathrm{JCR}$, and $\mathrm{CD}$ were major contributors in reviewing and providing feedback on the manuscript. All authors read and approved the final manuscript.

Acknowledgements - The authors would like to acknowledge Ms. Neera Bhatnagar and Mr. Jack Young, Librarians at the Health Sciences Library at McMaster University. Ms. Bhatnagar and Mr. Young shared their insight and expertise, assisting the authors to develop the search strategy, without receiving any funding or having assisted with the writing process.

\section{References}

1. Boyd H, McKernon S, Mullin B, Old A. Improving healthcare through the use of co-design. N Z Med J. 2012 Jun 29;125(1357):76-87. 
2. Slattery P, Saeri AK, Bragge P. Research co-design in health: a rapid overview of reviews. Health Research Policy and Systems. 2020 Feb $11 ; 18(1): 17$.

3. Batalden M, Batalden P, Margolis P, Seid M, Armstrong G, Opipari-Arrigan L, et al. Coproduction of healthcare service. BMJ Qual Saf. 2016 Jul 1;25(7):509-17.

4. Donetto S, Pierri P, Tsianakas V, Robert G. Experience-based Co-design and Healthcare Improvement: Realizing Participatory Design in the Public Sector. The Design Journal. 2015 Jun 1;18(2):227-48.

5. Frank L, Basch E, Selby JV, For the Patient-Centered Outcomes Research Institute. The PCORI Perspective on Patient-Centered Outcomes Research. JAMA. 2014 Oct 15;312(15):1513-4.

6. Booth FW, Roberts CK, Laye MJ. Lack of exercise is a major cause of chronic diseases. Compr Physiol. 2012 Apr;2(2):1143-211.

7. Kruk J. Physical activity in the prevention of the most frequent chronic diseases: an analysis of the recent evidence. Asian Pac J Cancer Prev. 2007 Sep;8(3):325-38.

8. Handschin C, Spiegelman BM. The role of exercise and PGC1a in inflammation and chronic disease. Nature. 2008 Jul;454(7203):463-9.

9. Ashe MC, Miller WC, Eng JJ, Noreau L. Older Adults, Chronic Disease and Leisure-Time Physical Activity. GER. 2009;55(1):64-72.

10. Paterson DH, Warburton DE. Physical activity and functional limitations in older adults: a systematic review related to Canada's Physical Activity Guidelines. International Journal of Behavioral Nutrition and Physical Activity. 2010 May 11;7(1):38.

11. Manini TM, Pahor M. Physical activity and maintaining physical function in older adults. British Journal of Sports Medicine. 2009 Jan 1;43(1):28-31.

12. Taylor D. Physical activity is medicine for older adults. Postgraduate Medical Journal. 2014 Jan 1;90(1059):26-32.

13. Thornton JS, Frémont P, Khan K, Poirier P, Fowles J, Wells GD, et al. Physical activity prescription: a critical opportunity to address a modifiable risk factor for the prevention and management of chronic disease: a position statement by the Canadian Academy of Sport and Exercise Medicine. Br J Sports Med. 2016 Sep;50(18):1109-14.

14. Caspersen CJ, Powell KE, Christenson GM. Physical activity, exercise, and physical fitness: definitions and distinctions for health-related research. Public Health Rep. 1985;100(2):126-31.

15. Bull FC, Al-Ansari SS, Biddle S, Borodulin K, Buman MP, Cardon G, et al. World Health Organization 2020 guidelines on physical activity and sedentary behaviour. Br J Sports Med. 2020 Dec 1;54(24):1451.

16. Langhammer B, Bergland A, Rydwik E. The Importance of Physical Activity Exercise among Older People. Biomed Res Int. 2018 Dec 5;2018:7856823.

17. More physical activity [Internet]. [cited 2021 Sep 6]. Available from: https://www.who.int/teams/health-workforce/world-directory-ofmedical-schools/health-promotion

18. Stockwell S, Trott M, Tully M, Shin J, Barnett Y, Butler L, et al. Changes in physical activity and sedentary behaviours from before to during the COVID-19 pandemic lockdown: a systematic review. BMJ Open Sport \& Exercise Medicine. 2021 Jan 1;7(1):e000960.

19. Browne RAV, Macêdo GAD, Cabral LLP, Oliveira GTA, Vivas A, Fontes EB, et al. Initial impact of the COVID-19 pandemic on physical activity and sedentary behavior in hypertensive older adults: An accelerometer-based analysis. Exp Gerontol. 2020 Dec;142:111121.

20. Cunningham C, O' Sullivan R, Caserotti P, Tully MA. Consequences of physical inactivity in older adults: A systematic review of reviews and meta-analyses. Scand J Med Sci Sports. 2020 May;30(5):816-27.

21. Goethals L, Barth N, Guyot J, Hupin D, Celarier T, Bongue B. Impact of Home Quarantine on Physical Activity Among Older Adults Living at Home During the COVID-19 Pandemic: Qualitative Interview Study. JMIR Aging. 2020 May 7;3(1):e19007.

22. Castañeda-Babarro A, Arbillaga-Etxarri A, Gutiérrez-Santamaría B, Coca A. Physical Activity Change during COVID-19 Confinement. International Journal of Environmental Research and Public Health. 2020 Jan;17(18):6878.

23. Meyer J, McDowell C, Lansing J, Brower C, Smith L, Tully M, et al. Changes in Physical Activity and Sedentary Behavior in Response to COVID-19 and Their Associations with Mental Health in 3052 US Adults. International Journal of Environmental Research and Public Health. 2020 Jan;17(18):6469.

24. Brett J, Staniszewska S, Mockford C, Herron-Marx S, Hughes J, Tysall C, et al. A Systematic Review of the Impact of Patient and Public Involvement on Service Users, Researchers and Communities. Patient. 2014 Dec 1;7(4):387-95.

25. Baldwin JN, Napier S, Neville S, Wright-St Clair VA. Impacts of older people's patient and public involvement in health and social care research: a systematic review. Age Ageing. 2018 Nov 1;47(6):801-9.

26. Schilling I, Gerhardus A. Methods for Involving Older People in Health Research-A Review of the Literature. Int J Environ Res Public Health. 2017 Nov 29;14(12):E1476.

27. Edward H, Constantin N, Ng H, Radisic A, D'Asti A, Yule A, et al. The use of co-design in developing physical activity interventions for older adults: a scoping review protocol. JBI. 2021 Forthcoming;

Page $9 / 13$ 
28. Levac D, Colquhoun H, O’Brien KK. Scoping studies: advancing the methodology. Implement Sci. 2010 Sep 20;5:69.

29. Peters M, Godfrey C, Mclnerney P, Munn Z, Trico A, Khalil H. Chapter 11: Scoping Reviews. In: Aromataris E, Munn Z, editors. JBI Manual for Evidence Synthesis [Internet]. JBI; 2020 [cited 2020 Dec 13]. Available from:

https://wiki.jbi.global/display/MANUAL/Chapter+11\%3A+Scoping+reviews

30. Arksey H, O'Malley L. Scoping studies: towards a methodological framework. International Journal of Social Research Methodology. 2005 Feb 1;8(1):19-32.

31. Tricco AC, Lillie E, Zarin W, O’Brien KK, Colquhoun H, Levac D, et al. PRISMA Extension for Scoping Reviews (PRISMA-ScR): Checklist and Explanation. Ann Intern Med. 2018 Oct 2;169(7):467.

32. McGowan J, Sampson M, Salzwedel DM, Cogo E, Foerster V, Lefebvre C. PRESS Peer Review of Electronic Search Strategies: 2015 Guideline Statement. J Clin Epidemiol. 2016 Jul;75:40-6.

33. Viera AJ, Garrett JM. Understanding interobserver agreement: the kappa statistic. Fam Med. 2005 May;37(5):360-3.

34. Sheridan S, Schrandt S, Forsythe L, Hilliard TS, Paez KA, Advisory Panel on Patient Engagement (2013 inaugural panel). The PCORI Engagement Rubric: Promising Practices for Partnering in Research. Ann Fam Med. 2017 Mar;15(2):165-70.

35. Boote J, Baird W, Sutton A. Public involvement in the systematic review process in health and social care: A narrative review of case examples. Health Policy. 2011 Oct 1;102(2):105-16.

36. Leask CF, Sandlund M, Skelton DA, Altenburg TM, Cardon G, Chinapaw MJM, et al. Framework, principles and recommendations for utilising participatory methodologies in the co-creation and evaluation of public health interventions. Research Involvement and Engagement. 2019 Jan 9;5(1):2.

37. Moher D, Liberati A, Tetzlaff J, Altman DG. Preferred reporting items for systematic reviews and meta-analyses: the PRISMA statement. BMJ. 2009 Jul 21;339:b2535.

38. Brox E, Konstantinidis ST, Evertsen G. User-Centered Design of Serious Games for Older Adults Following 3 Years of Experience With Exergames for Seniors: A Study Design. JMIR Serious Games [Internet]. 2017 Jan 11 [cited 2021 Jun 5];5(1). Available from: https://www.ncbi.nlm.nih.gov/pmc/articles/PMC5266825/

39. Castro PC, Romano LB, Frohlich D, Lorenzi LJ, Campos LB, Paixão A, et al. Tailoring digital apps to support active ageing in a low income community. PLOS ONE. 2020 Dec 10;15(12):e0242192.

40. Da Silva Júnior JLA, Biduski D, Bellei EA, Becker OHC, Daroit L, Pasqualotti A, et al. A Bowling Exergame to Improve Functional Capacity in Older Adults: Co-Design, Development, and Testing to Compare the Progress of Playing Alone Versus Playing With Peers. JMIR Serious Games [Internet]. 2021 Jan 29 [cited 2021 Jun 6];9(1). Available from: https://www.ncbi.nlm.nih.gov/pmc/articles/PMC7880815/

41. Giné-Garriga M, Sandlund M, Dall PM, Chastin SFM, Pérez S, Skelton DA. A Novel Approach to Reduce Sedentary Behaviour in Care Home Residents: The GET READY Study Utilising Service-Learning and Co-Creation. Int J Environ Res Public Health [Internet]. 2019 Feb [cited 2021 Jun 6];16(3). Available from: https://www.ncbi.nlm.nih.gov/pmc/articles/PMC6388363/

42. Giné-Garriga M, Dall PM, Sandlund M, Jerez-Roig J, Chastin SFM, Skelton DA. A Pilot Randomised Clinical Trial of a Novel Approach to Reduce Sedentary Behaviour in Care Home Residents: Feasibility and Preliminary Effects of the GET READY Study. Int J Environ Res Public Health [Internet]. 2020 Apr [cited 2021 Jun 6];17(8). Available from: https://www.ncbi.nlm.nih.gov/pmc/articles/PMC7215704/

43. Lacey G, MacNamara S. User involvement in the design and evaluation of a smart mobility aid. J Rehabil Res Dev. 2000 Dec;37(6):70923.

44. Lu Y, Valk C, Steenbakkers J, Bekker T, Visser T, Proctor G, et al. Can technology adoption for older adults be co-created? Gerontechnology. 2017 Nov 7;16:151-9.

45. Mansson L, Wiklund M, Öhberg F, Danielsson K, Sandlund M. Co-Creation with Older Adults to Improve User-Experience of a Smartphone Self-Test Application to Assess Balance Function. Int J Environ Res Public Health [Internet]. 2020 Jun [cited 2021 Jun 6];17(11). Available from: https://www.ncbi.nlm.nih.gov/pmc/articles/PMC7312460/

46. Parker SJ, Chen EK, Pillemer K, Filiberto D, Laureano E, Piper J, et al. Participatory Adaptation of an Evidence-Based, Arthritis SelfManagement Program: Making Changes to Improve Program Fit. Fam Community Health. 2012;35(3):236-45.

47. Revenäs Å, Johansson A-C, Ehn M. Integrating Key User Characteristics in User-Centered Design of Digital Support Systems for Seniors' Physical Activity Interventions to Prevent Falls: Protocol for a Usability Study. JMIR Res Protoc [Internet]. 2020 Dec 21 [cited 2021 Jun 6];9(12). Available from: https://www.ncbi.nlm.nih.gov/pmc/articles/PMC7781794/

48. Swallow D, Petrie H, Power C, Lewis A, Edwards ADN. Involving Older Adults in the Technology Design Process: A Case Study on Mobility and Wellbeing in the Built Environment. Stud Health Technol Inform. 2016;229:615-23.

49. Timmerman JG, Tönis TM, Dekker-van Weering MGH, Stuiver MM, Wouters MWJM, van Harten WH, et al. Co-creation of an ICT-supported cancer rehabilitation application for resected lung cancer survivors: design and evaluation. BMC Health Services Research. 2016 Apr 27;16(1):155. 
50. Walsh DMJ, Moran K, Cornelissen V, Buys R, Claes J, Zampognaro P, et al. The development and codesign of the PATHway intervention: a theory-driven eHealth platform for the self-management of cardiovascular disease. Transl Behav Med. 2019 Jan 1;9(1):76-98.

51. Giné-Garriga M, Sandlund M, Dall PM, Chastin SFM, Pérez S, Skelton DA. A co-created intervention with care home residents and university students following a service-learning methodology to reduce sedentary behaviour: The GET READY project protocol. J Frailty Sarcopenia Falls. 2018 Sep;3(3):132-7.

52. Bird M-L, Mortenson BW, Chu F, Acerra N, Bagnall E, Wright A, et al. Building a Bridge to the Community: An Integrated Knowledge Translation Approach to Improving Participation in Community-Based Exercise for People After Stroke. Phys Ther. 2019 Mar;99(3):28696.

53. Frigault JS, Giles AR. Culturally Safe Falls Prevention Program for Inuvialuit Elders in Inuvik, Northwest Territories, Canada: Considerations for Development and Implementation. Canadian Journal on Aging / La Revue canadienne du vieillissement. 2020 Jun;39(2):190-205.

54. Leask CF, Colledge N, Laventure RME, McCann DA, Skelton DA. Co-Creating Recommendations to Redesign and Promote Strength and Balance Service Provision. Int J Environ Res Public Health [Internet]. 2019 Sep [cited 2021 Jun 6];16(17). Available from: https://www.ncbi.nlm.nih.gov/pmc/articles/PMC6747106/

55. Leask CF, Sandlund M, Skelton DA, Chastin SF. Co-creating a tailored public health intervention to reduce older adults' sedentary behaviour. Health Education Journal. 2017 Aug 1;76(5):595-608.

56. Seino S, Kitamura A, Tomine Y, Tanaka I, Nishi M, Nonaka K, et al. A Community-Wide Intervention Trial for Preventing and Reducing Frailty Among Older Adults Living in Metropolitan Areas: Design and Baseline Survey for a Study Integrating Participatory Action Research With a Cluster Trial. J Epidemiol. 2019 Feb 5;29(2):73-81.

57. Verhoeven F, Cremers A, Schoone M, Dijk J van. Mobiles for mobility: Participatory design of a 'Happy walker' that stimulates mobility among older people. GERONTOLOGY. 2016;15(1):32-44.

58. Davies J, Lester C, O’Neill M, Williams G. Sustainable participation in regular exercise amongst older people: Developing an action research approach. Health Education Journal. 2008 Mar 1;67(1):45-55.

59. Bammann K, Recke C, Albrecht BM, Stalling I, Doerwald F. Promoting Physical Activity Among Older Adults Using Community-Based Participatory Research With an Adapted PRECEDE-PROCEED Model Approach: The AEQUIPA/OUTDOOR ACTIVE Project. Am J Health Promot. 2021 Mar 1;35(3):409-20.

60. Bammann K, Drell C, Lübs LL, Stalling I. Cluster-randomised trial on participatory community-based outdoor physical activity promotion programs in adults aged 65-75 years in Germany: protocol of the OUTDOOR ACTIVE intervention trial. BMC Public Health [Internet]. 2018 Oct 23 [cited 2021 Jun 6];18. Available from: https://www.ncbi.nlm.nih.gov/pmc/articles/PMC6199784/

61. Brach JS, Perera S, Gilmore S, VanSwearingen JM, Brodine D, Wert D, et al. Stakeholder involvement in the design of a patient-centered comparative effectiveness trial of the "On the Move" group exercise program in community-dwelling older adults. Contemp Clin Trials. 2016 Sep;50:135-42.

62. Arnautovska U, O'callaghan F, Hamilton K. Behaviour change techniques to facilitate physical activity in older adults: what and how. Ageing \& Society. 2018 Dec;38(12):2590-616.

63. Balbale S, Schwingel A, Chodzko-Zajko W, Huhman M. Visual and Participatory Research Methods for the Development of Health Messages for Underserved Populations. Health communication. 2013 Oct 30;29.

64. Åberg AC, Halvorsen K, From I, Bruhn ÅB, Oestreicher L, Melander-Wikman A. A Study Protocol for Applying User Participation and CoLearning-Lessons Learned from the eBalance Project. Int J Environ Res Public Health. 2017 May 10;14(5).

65. Pearson J, Walsh N, Carter D, Koskela S, Hurley M. Developing a Web-Based Version of An Exercise-Based Rehabilitation Program for People With Chronic Knee and Hip Pain: A Mixed Methods Study. JMIR Res Protoc [Internet]. 2016 May 19 [cited 2021 Jun 6];5(2). Available from: https://www.ncbi.nlm.nih.gov/pmc/articles/PMC4891573/

66. Tabak M, de Vette F, van Dijk H, Vollenbroek-Hutten M. A Game-Based, Physical Activity Coaching Application for Older Adults: Design Approach and User Experience in Daily Life. Games Health J. 2020 Jun;9(3):215-26.

67. Kittipimpanon K, Amnatsatsue K, Kerdmongkol P, Maruo SJ, Nityasuddhi D. Development and Evaluation of a Community-based Fall Prevention Program for Elderly Thais. Pacific Rim International Journal of Nursing Research. 2012;16(3):222-35.

68. Jitramontree N, Chatchaisucha S, Thaweeboon T, Kutintara B, Intanasak S. Action Research Development of a Fall Prevention Program for Thai Community-dwelling Older Persons. Pacific Rim International Journal of Nursing Research. 2015 Feb 10;19(1):69-79.

69. Szanton SL, Walker RK, Lim JH, Fisher L, Zhan A, Gitlin LN, et al. Development of an Exergame for Urban-dwelling Older Adults With Functional Limitations: Results and Lessons Learned. Prog Community Health Partnersh. 2016;10(1):73-81.

70. Browne J, Medenblik A, Pebole M, Gregg JJ, Hall KS. Qualitative Analysis of a Supervised Exercise Program for Older Veterans With PTSD. The American Journal of Geriatric Psychiatry. 2021 Jun 1;29(6):565-72. 
71. Visual and participatory research methods for the development of health messages for underserved populations - PubMed [Internet]. [cited 2021 Jun 5]. Available from: https://pubmed.ncbi.nlm.nih.gov/24171509/

72. Drahota A, Meza RD, Brikho B, Naaf M, Estabillo JA, Gomez ED, et al. Community-Academic Partnerships: A Systematic Review of the State of the Literature and Recommendations for Future Research. Milbank Q. 2016 Mar;94(1):163-214.

73. Cukor D, Cohen LM, Cope EL, Ghahramani N, Hedayati SS, Hynes DM, et al. Patient and Other Stakeholder Engagement in Patient-Centered Outcomes Research Institute Funded Studies of Patients with Kidney Diseases. Clin J Am Soc Nephrol. 2016 Sep 7;11(9):1703-12.

74. Esmail L, Moore E, Rein A. Evaluating patient and stakeholder engagement in research: moving from theory to practice. J Comp Eff Res. 2015 Mar;4(2):133-45.

75. Birt L, Scott S, Cavers D, Campbell C, Walter F. Member Checking: A Tool to Enhance Trustworthiness or Merely a Nod to Validation? Qual Health Res. 2016 Nov 1;26(13):1802-11.

76. Torrance H. Triangulation, Respondent Validation, and Democratic Participation in Mixed Methods Research. Journal of Mixed Methods Research. 2012 Apr 1;6(2):111-23.

77. Carroll C, Patterson M, Wood S, Booth A, Rick J, Balain S. A conceptual framework for implementation fidelity. Implement Sci. 2007 Nov $30 ; 2: 40$.

\section{Figures}

\section{Use of Co-Design Terminology}

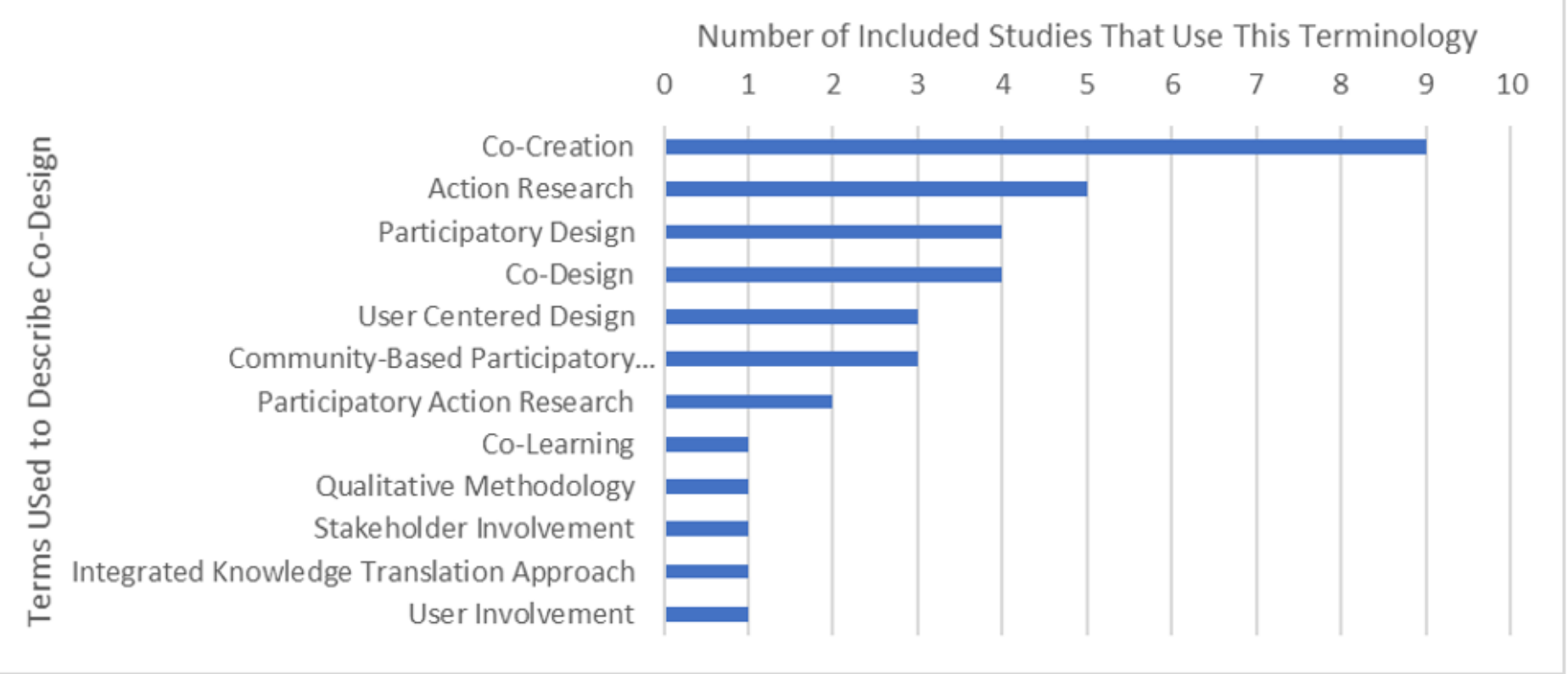

Figure 1

Use of Co-Design Terminology in Included Studies 


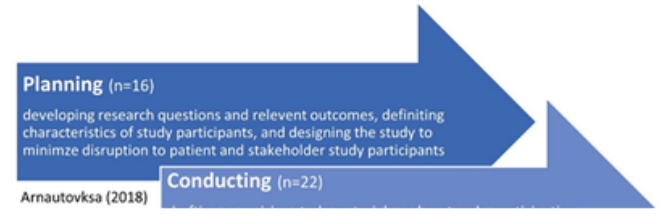

\section{Figure 2}

\section{Distribution of studies according to phase of research in which end-users were involved.}

\section{Determine the levels of involvement of the end-users in the included studies}

The levels of involvement of end-users progress from consultation to collaboration to consumer control the highest level involvement. Most end-users were involved at either the consultation (52\%) or collaboration (45\%) level in the included studies. Figure $2 \mathrm{~b}$ summarizes the different levels of involvement, the number of studies within each level, and the methods used within each level.

\section{Levels of involvement of end-users in the co-design process.}

\section{Measuring Success and/or Effectiveness of Co-Design}

None of the included studies described any type of process to evaluate the success and/or effectiveness of the co-design process itself. However, some studies evaluated the success and/or effectiveness of the co-design process through indirect methods, such as measuring participant satisfaction of the PA intervention itself $(n=4)$, intervention adherence measures $(n=6)$, and by assessing changes in PA performance and levels of PA $(n=5)$ (Table 2). Of the 15 studies that indirectly evaluated co-design, all reported positive trial outcomes that they attribute to the use of a co-design process.

\section{Supplementary Files}

This is a list of supplementary files associated with this preprint. Click to download.

- AdditionalFile1PRISMAChecklist.docx

- AdditionalFile2SearchStrategy.docx

- AdditionalFile3PRISMAFlowChart.docx

- AdditionalFile4ReferenceListofExcludedStudies.docx

- AdditionalFile5StudyCharacteristics.xlsx

- AdditionalFile60perationalDefinitions.docx 